\title{
Multiple redundant stress resistance mechanisms are induced in Salmonella enterica serovar Typhimurium in response to alteration of the intracellular environment via TLR4 signalling
}

\section{Correspondence \\ Clare E. Bryant \\ ceb27@cam.ac.uk}

Received 28 April 2009

Accepted 12 June 2009
John A. Wright, ${ }^{1} \dagger \ddagger$ Sabine S. Tötemeyer, ${ }^{1} \dagger \S$ Isabelle Hautefort, ${ }^{2}$ Corinne Appia-Ayme, ${ }^{2}$ Mark Alston, ${ }^{2}$ Vittoria Danino, ${ }^{2}$ Gavin K. Paterson, ${ }^{1}$ Pietro Mastroeni, ${ }_{1}^{1}$ Nathalie Ménager, ${ }^{1}$ Matthew Rolfe, ${ }^{2}$ Arthur Thompson, ${ }^{2}$ Sanja Ugrinovic, ${ }^{1}$ Leanne Sait, ${ }^{3}$ Tom Humphrey, ${ }^{3}$ Helen Northen, ${ }^{1}$ Sarah E. Peters, ${ }^{1}$ Duncan J. Maskell, ${ }^{1}$ Jay C. D. Hinton $^{2} \|$ and Clare E. Bryant ${ }^{1}$

\footnotetext{
${ }^{1}$ Department of Veterinary Medicine, University of Cambridge, Madingley Road, Cambridge CB3 OES, UK

${ }^{2}$ Molecular Microbiology Group, Institute of Food Research, Norwich NR4 7UA, UK

${ }^{3}$ School of Clinical Veterinary Sciences, University of Bristol, Langford House, Langford, Bristol BS40 5DU, UK
}

Toll-like receptor 4 (TLR4) senses bacterial LPS and is required for the control of systemic Salmonella enterica serovar Typhimurium infection in mice. The mechanisms of TLR4 activation and its downstream signalling cascades are well described, yet the direct effects on the pathogen of signalling via this receptor remain unknown. To investigate this we used microarray-based transcriptome profiling of intracellular $S$. Typhimurium during infection of primary bone marrowderived macrophages from wild-type and TLR4-deficient mice. We identified $17 \mathrm{~S}$. Typhimurium genes that were upregulated in the presence of functional TLR4. Nine of these genes have putative functions in oxidative stress resistance. We therefore examined $S$. Typhimurium gene expression during infection of NADPH oxidase-deficient macrophages, which lack normal oxidative killing mechanisms. We identified significant overlap between the 'TLR4-responsive' and 'NADPH oxidase-responsive' genes. This is new evidence for a link between TLR4 signalling and NADPH oxidase activity. Interestingly, with the exception of a dps mutant, $S$. Typhimurium strains lacking individual TLR4- and/or oxidative stress-responsive genes were not attenuated during intravenous murine infections. Our study shows that TLR4 activity, either directly or indirectly, induces the expression of multiple stress resistance genes during the intracellular life of $S$. Typhimurium.

†These authors contributed equally to this work.

łPresent address: Division of Microbial Diseases, UCL Eastman Dental Institute, University College London, 256 Gray’s Inn Road, London WC1X 8LD, UK.

§Present address: School of Veterinary Medicine and Science, University of Nottingham, Sutton Bonington Campus, Loughborough LE12 5RD, UK. ||Present address: School of Genetics and Microbiology, Moyne Institute of Preventive Medicine, Trinity College, Dublin 2, Ireland.

Abbreviations: BMDM, bone marrow-derived macrophage; FDR, false discovery rate; SPI, Salmonella pathogenicity island; TLR, Toll-like receptor. The ArrayExpress accession numbers for the complete $\mathrm{gp} 91^{-/}, \mathrm{TLR} 4^{-{ }^{-}}$and hydrogen peroxide datasets associated with this paper are E-MEXP1089 and E-MEXP-1067.

Five supplementary tables are available with the online version of this paper. 


\section{INTRODUCTION}

Salmonella enterica serovar Typhimurium (S. Typhimurium) causes a severe systemic disease in mice, which shares many features with invasive salmonelloses of other mammals. Murine infection by $S$. Typhimurium has been extensively investigated as a model for human typhoid fever caused by serovar Typhi (Santos et al., 2001). A crucial aspect of the pathogenicity of invasive Salmonella infections is the ability of the pathogen to invade and survive within phagocytes (Fields et al., 1986). Following phagocytosis of a pathogen by a macrophage, and its compartmentalization into a phagosome, a sequence of antibacterial processes is initiated in the host cell. These include superoxide production, catalysed by NADPH oxidase, and nitric oxide production, catalysed by inducible nitric oxide synthase, acidification of the phagosome through the action of the vacuolar ATPase, and fusion with lysosomes to deliver hydrolases and cationic antimicrobial peptides. These processes generate a highly bactericidal environment within the phagosome (Steele-Mortimer, 2008; Haas, 2007). Following internalization S. Typhimurium becomes compartmentalized within a phagosome which fails to completely mature, known as the Salmonella-containing vacuole (SCV). S. Typhimurium subverts the phagosomal maturation pathway through prevention or delay of fusion of selected endosomes harbouring antimicrobial components with the SCV (Steele-Mortimer, 2008; McCollister \& Vazquez-Torres, 2006). Effectors delivered by type III secretion systems encoded by Salmonella pathogenicity islands (SPI) 1 and 2 are involved in regulating SCV biogenesis, with SPI2 effectors being required for intra-macrophage replication and systemic infection of mice (Waterman \& Holden, 2003). However, knowledge of the molecular details of the exact actions of SPI effectors, and the extent to which individual effector proteins subvert the phagosomal pathway, is currently limited (Steele-Mortimer, 2008).

During infection, the cellular components of bacteria are recognized by the innate immune system via specific ligand-receptor binding. This involves host cell pattern recognition receptors including Toll-like receptors (TLRs) (Akira et al., 2006). There are 12 known TLRs across different species, which are capable of binding to specific pathogen-associated molecular patterns (Akira et al., 2006). One of these is TLR4, which is a key component of the innate immune response to Gram-negative bacteria (Takeuchi et al., 1999; Lembo et al., 2003) and is critical in controlling $S$. Typhimurium infections in mice (Weiss et al., 2004). Mice lacking active TLR4 are unable to suppress bacterial growth in the spleen and liver, and exhibit substantially reduced survival when experimentally infected with S. Typhimurium (O’Brien et al., 1980; VazquezTorres et al., 2004; Eisenstein et al., 1982). TLR4 recognizes the lipid A portion of bacterial LPS through cooperation with three murine accessory proteins: LPS-binding protein (LBP), CD14 and MD-2 (Teghanemt et al., 2007; Kim et al., 2007).
Infection of macrophages with $S$. Typhimurium induces a similar pattern of changes in host inflammatory response and antimicrobial gene expression to that seen when macrophages are stimulated by LPS (Rosenberger et al., 2000). Thus it is likely that during Salmonella infection it is predominantly LPS that activates the macrophage inflammatory response, establishing production of inflammatory cytokines such as TNF- $\alpha$ and antibacterial mediators such as nitric oxide (Rosenberger et al., 2000; Royle et al., 2003). In addition, TLR4 and the phagosomal NADPH oxidase are functionally linked: the oxidative burst is elicited in monocytes in response to LPS in a TLR4-dependent manner (Ryan et al., 2004) and LPS stimulation activates NADPH oxidase via IRAK-4 (Pacquelet et al., 2007).

The host cell response to $S$. Typhimurium infection is increasingly well understood, but the responses of the infecting salmonellae to the changing macrophage environment remain poorly characterized. Changes in bacterial gene expression may occur as a consequence of detecting the hostile intracellular environment generated by the host response to infection. It is important therefore to understand how the bacteria respond to changes in the host cell environment. Transcriptome profiling of $S$. Typhimurium during infection of J774-A.1 macrophagelike cells, and of $S$. Typhi in THP-1 macrophages, has revealed large changes in gene expression compared to in vitro growth, giving an indication of how $S$. enterica responds to the intracellular environment (Eriksson et al., 2003; Faucher et al., 2006).

In this study we used primary bone marrow-derived macrophages (BMDMs) from knockout mice deficient in either TLR4 or gp91 NADPH oxidase to determine whether the alteration of the intracellular environment by these key components of the innate immune system in turn leads to gene expression changes in $S$. Typhimurium living inside these cells. We discovered that there were changes in the expression of many $S$. Typhimurium genes involved in resistance to the oxidative burst following TLR4 signalling. The fact that the deletion of any one of these genes individually does not cause attenuation in murine infection models suggests that $S$. Typhimurium possesses redundant mechanisms that facilitate resistance to the initial innate immune response and allow subsequent persistence needed to establish systemic infection.

\section{METHODS}

Mice. C57BL/6 mice were supplied by Harlan UK. TLR4 ${ }^{-1-}$ mice (kindly supplied by Shizuo Akira: Hoshino et al., 1999) and gp91-1knockout mice (lacking NADPH oxidase activity: Pollock et al., 1995) were bred at the University of Cambridge under Specific Pathogen Free conditions.

Bacterial strains, media and reagents. The $S$. Typhimurium strain SL1344 (Hoiseth \& Stocker, 1981) and its parental strain 4/74 (Jones et al., 1991; Rankin \& Taylor, 1966) were used in this study. These two strains only differ in the hisG mutation carried by SL1344. Both strains are virulent in a variety of animals (Villarreal-Ramos et al., 
2000; Paulin et al., 2007). S. Typhimurium was grown in LuriaBertani (LB) broth or on LB agar at $37^{\circ} \mathrm{C}$. The bacterial strains used in this study are summarized in Table 1.

Infection of bone marrow macrophages and isolation of bacterial RNA. Primary BMDMs were isolated from femurs and tibiae of mice killed by cervical dislocation. Briefly, the bone marrow was flushed out with RPMI 1640 medium supplemented with $10 \%$ $(\mathrm{v} / \mathrm{v})$ fetal calf serum, $2 \mathrm{mM}$ glutamine, $5 \%(\mathrm{v} / \mathrm{v})$ horse serum and $1 \mathrm{mM}$ sodium pyruvate, then plated onto Petri dishes. For maintenance of the BMDMs in culture this medium was further supplemented with $20 \%(\mathrm{v} / \mathrm{v})$ of supernatant taken from L929 cells (a murine M-CSF-producing cell line) (Royle et al., 2003). For infection experiments leading to RNA isolation, BMDMs were plated onto 6well plates at a density of $2 \times 10^{6}$ cells per well and infected with $S$. Typhimurium SL1344 (opsonized for $30 \mathrm{~min}$ at $37{ }^{\circ} \mathrm{C}$ with $10 \%, \mathrm{v} / \mathrm{v}$, normal mouse serum) at an m.o.i. of 200:1. To minimize SPI1 expression, which could lead to macrophage apoptosis (Monack et al., 1996), bacteria were grown overnight on LB plates at $37^{\circ} \mathrm{C}$ and suspended in PBS before opsonization (Eriksson et al., 2003). After $1 \mathrm{~h}$ of phagocytosis, extracellular bacteria were killed with $30 \mu \mathrm{g}$ gentamicin $\mathrm{ml}^{-1}$ for $1 \mathrm{~h}$ followed by further incubation with $5 \mu \mathrm{g}$ gentamicin $\mathrm{ml}^{-1}$.

RNA extraction. At $2 \mathrm{~h}$ or $4 \mathrm{~h}$ post-infection, infected macrophages were lysed on ice for $30 \mathrm{~min}$ in $0.1 \%$ SDS, $1 \%$ acidic phenol and $19 \%$ ethanol in water as described previously (Eriksson et al., 2003; Hinton et al., 2004). S. Typhimurium cells were pelleted after centrifugation and RNA was prepared using the Promega SV and Ambion RiboPure-Bacteria total RNA purification kits. Bacterial RNA was further purified by phenol/chloroform extraction. Each sample yielded 5-10 $\mu \mathrm{g}$ of total bacterial RNA.

Peroxide stress conditions for transcriptomic analysis. A $25 \mathrm{ml}$ overnight culture of $S$. Typhimurium 4/74 was diluted 1000-fold in flasks containing $25 \mathrm{ml} \mathrm{LB}$ broth and grown to $\mathrm{OD}_{600} 0.1$. Hydrogen peroxide was added to half of the flasks at a final concentration of $1 \mathrm{mM}$ and the other half were used as controls. The cultures were allowed to grow for a further $12 \mathrm{~min}$ before samples were taken for transcriptomic analysis (Nagy et al., 2006). Triplicate biological replicates were performed (Porwollik et al., 2003).

Microarray procedures. Transcriptomic techniques involved $S$. Typhimurium microarrays constructed at the Institute of Food
Research, Norwich, UK, as described previously (Nagy et al., 2006; Lucchini et al., 2005). All transcriptomic analyses were defined using microarrays covering 4193 genes, $92 \%$ of which are common between S. Typhimurium strains LT2 and SL1344 (Kelly et al., 2004).

Probe preparation and scanning. The yield of bacterial RNA extracted from infected BMDMs was relatively low; therefore RNA was first reverse-transcribed into cDNA and subsequently labelled by random priming with the Klenow fragment of DNA polymerase I according to the 'Labelling protocol for reduced amounts of RNA' described at http://www.ifr.ac.uk/Safety/MolMicro/protocols.html. Fluorescently labelled genomic DNA was used as a reference channel for each experiment. Slides were scanned on an Axon 4000B scanner (Axon Instruments) using GenePix version 1.4 software (Axon Instruments). Each hybridization was performed twice. Two biological replicates were performed for the wild-type and TLR4 ${ }^{-1-}$ BMDM, and three for the gp91 ${ }^{-/-}$BMDM.

Data analysis. Data analysis was performed as described by Eriksson-Ygberg et al. (2006). Spots showing a reference signal lower than background plus two standard deviations, or obvious blemishes, were excluded from subsequent analyses. Local background was subtracted from spot signals, and fluorescence ratios were calculated. To compensate for unequal dye incorporation or any effect of the amount of template, data centring was performed by bringing the median natural logarithm of the ratios for each group of spots printed by the same pin to zero. In a few cases, when comparing results from different hybridizations, we observed slight deviations which were dependent on gene expression levels. These were corrected using the Loess function in R (Limma package). Differentially expressed genes were identified by a Perl implementation of the rank products (RP) method (Breitling et al., 2004), which performs well with limited numbers of samples or datasets that exhibit some biological noise (Jeffery et al., 2006). The log fold-changes for all pair-wise comparisons between the two sample classes (BMDM and gp91 ${ }^{-1-}$ BMDM, or BMDM and TLR4 $4^{-1-}$ BMDM) were used to calculate the $\mathrm{RP}$ values. The false discovery rate (FDR) for each gene was determined by using 1000 random permutations of the data to assign significance to those RP values. This was only used to analyse the TLR4 and NADPH oxidase data. Forty-nine and 36 genes were upand downregulated, respectively, in wild-type BMDM compared with gp91 ${ }^{-1-}$ BMDM samples, and 44 and 28 genes up- and downregulated, respectively, in wild-type BMDM compared with TLR4 ${ }^{-1-}$ BMDM samples, with significance defined as an FDR of $\leqslant 1 \%$. The

Table 1. S. Typhimurium strains used in this study

\begin{tabular}{|c|c|c|}
\hline Strain & Genotype or relevant characteristics & Source or reference \\
\hline $4 / 74$ & Virulent for mice & Jones et al. (1991); Rankin \& Taylor (1966) \\
\hline SL1344 & 4/74 hisG46 virulent mouse strain & Hoiseth \& Stocker (1981) \\
\hline$\Delta S T M 0561$ & SL1344 $\triangle S T M 0561::$ cat & This study \\
\hline$\Delta S T M 1485$ & SL1344 $\triangle S T M 1485:$ : kan & This study \\
\hline$\Delta o x y S$ & SL1344 $\Delta$ oxyS: : kan & This study \\
\hline$\triangle a h p C F$ & SL1344 $\Delta a h p C a h p F::$ kan & This study \\
\hline$\Delta d p s$ & SL1344 $\Delta d p s:$ : cat & This study \\
\hline$\Delta a h p C F \Delta k a t G$ & SL1344 $\Delta a h p C a h p F:$ : kan $\Delta k a t G:$ : cat & This study \\
\hline$\Delta g r x A$ & SL1344 $\Delta g r x A::$ cat & This study \\
\hline$\Delta k a t G$ & SL1344 $\Delta k a t G::$ cat & This study \\
\hline$\triangle s t c A B C D$ & SL1344 $\Delta$ stcAstcBstcCstcF: : cat & This study \\
\hline$\Delta s u f A$ & SL1344 $\Delta s u f A:$ : cat & This study \\
\hline$\triangle s u f A B C D S E$ & SL1344 $\Delta$ sufAsufBsufCsufDsufSsufE: : cat & This study \\
\hline$\Delta y e h E$ & SL1344 yehE::kan & This study \\
\hline
\end{tabular}


complete dataset is available as supplementary material with the online version of this paper (Supplementary Tables S1 and S2). For the hydrogen peroxide data, an ANOVA test with a Benjamini and Hochberg FDR of 0.05 was used to identify genes that were significantly differentially expressed by strain $4 / 74$ between LB and LB plus peroxide. Data that passed the quality controls were analysed using Genespring version GX7.3 software (Agilent). The complete gp91 ${ }^{-1-}, \mathrm{TLR}^{-1-}$ and hydrogen peroxide datasets have been submitted to ArrayExpress with the accession numbers E-MEXP1089 and E-MEXP-1067.

Construction of S. Typhimurium mutants. Chromosomal deletion mutants of $S$. Typhimurium SL1344 were constructed using the onestep gene-disruption technique (Datsenko \& Wanner, 2000; Mo et al., 2006). Primers listed in Supplementary Table S3 were used to amplify gene cassettes encoding resistance to chloramphenicol (from $\mathrm{pKD} 3$ or pACYC184) or kanamycin (from pKD4 or pACYC177) with the addition of $5^{\prime}$ and $3^{\prime}$ homology arms complementary to the flanking regions of the chromosomal gene to be deleted at either end of the appropriate resistance cassette. SL1344 was transformed with pBAD $\lambda$ red carrying the phage lambda genes exo, bet and gam, induced with $0.2 \%(\mathrm{w} / \mathrm{v}$, final concentration) arabinose, followed by electroporation of the mutagenic PCR products and selection with the appropriate antibiotic. Allelic replacement was verified by a test PCR using primers listed in Supplementary Table S4. After DNA sequencing to confirm the sequence at the junction of the antibiotic resistance cassette and the disrupted gene, the mutations were moved by P22 bacteriophage transduction (Schmieger, 1972) into a clean SL1344 background and verified by colony PCR, or alternatively cured of pBAD $\lambda$ red by serial passage, and loss of plasmid confirmed by testing for ampicillin resistance and by attempted plasmid purification.

Infection of mice. Bacteria were grown overnight at $37^{\circ} \mathrm{C}$ as a static culture in LB medium, then washed and diluted in PBS to obtain $5 \times 10^{3}$ c.f.u. $\mathrm{ml}^{-1}$. Aliquots $\left(200 \mu \mathrm{l}, 10^{3}\right.$ c.f.u. $)$ of this bacterial suspension were injected into the tail veins of six- to eight-week-old mice. Appropriate dilutions of the inoculum were plated on LB agar for enumeration of the number of viable bacteria given to the mice. At each time point post-infection, four mice were killed by cervical dislocation and spleens and livers were removed. The organs were placed into $10 \mathrm{ml}$ sterile distilled water and homogenized in a Colworth stomacher for subsequent determination of viable bacterial counts by plating out appropriate dilutions of the homogenates on LB agar.

\section{RESULTS}

\section{Signalling via TLR4 leads to the differential expression of more than $\mathbf{7 0}$ genes in intracellular S. Typhimurium}

Previous studies have shown that signalling via TLR4 is required for efficient killing of intracellular bacteria (Vazquez-Torres et al., 2004). We used a transcriptomic approach to determine the impact of TLR4 activation upon salmonellae infecting macrophages in culture. We compared the gene expression profiles of intracellular $S$. Typhimurium infecting BMDMs from either wild-type or $\mathrm{TLR}^{-/-}$mice. Expression levels of $72 \mathrm{~S}$. Typhimurium genes were significantly different when infections in wildtype BMDMs were compared to those in TLR4 $4^{-1-}$ BMDMs at a false discovery rate (FDR) of 0.01 , suggesting that these genes are specifically differentially regulated in response to TLR4 signalling (Supplementary Table S1). Forty-four of these genes were expressed at higher levels and 28 of them were expressed at lower levels in wild-type macrophages when compared to the TLR4-deficient cells. The list of differentially expressed genes was limited further by the application of a stringent fold-change cut-off of 3 , in order to focus solely on genes with substantially different expression levels during infection of the wild-type and TLR4 $^{-1-}$ BMDMs. The expression of 17 genes was altered more than 3-fold, and all of these genes were more highly expressed in the wild-type compared to the TLR4 ${ }^{-l-}$ BMDMs (Fig. 1a, Supplementary Table S1). For convenience we designate these as 'TLR4-inducible' $S$. Typhimurium genes. A number of these protect the bacterium from oxidative damage in vitro, including kat $G$ (catalase), ahpCF (alkyl hydroperoxide reductase), dps (DNA-binding protein in stationary phase) (Janssen $e t$ al., 2003) and sufABCDSE (repair of oxidatively damaged [Fe-S] clusters) (Nachin et al., 2003). Infection studies have shown that $d p s$, for example, is required for bacterial survival inside macrophages and for full virulence of $S$. Typhimurium in mice (Halsey et al., 2004).

\section{Many 'TLR4-inducible' genes are also induced in response to the oxidative burst}

Since a substantial number of the 'TLR4-inducible' genes are involved in oxidative stress resistance, we compared $S$. Typhimurium gene expression during infection of BMDMs isolated from wild-type mice or gp91 ${ }^{-1-}$ knockout mice that lack functional NADPH oxidase. Expression levels of $85 \mathrm{~S}$. Typhimurium genes were significantly different when infections in wild-type BMDMs were compared to those in gp91 ${ }^{-1-}$ BMDMs at an FDR of 0.01, suggesting that these genes are specifically differentially regulated in response to NADPH oxidase activity (Supplementary Table S2). Fortynine of these genes were expressed at higher levels and 36 of them were expressed at lower levels in wild-type macrophages when compared to the NADPH oxidase-deficient cells. When the more stringent 3-fold cut-off was applied, 21 genes were expressed at higher levels and 13 at lower levels increased in the presence of NADPH oxidase. We designated these 34 genes as 'NADPH oxidase-responsive', with the 21 genes that were more highly expressed in wildtype BMDMs designated 'NADPH oxidase-inducible'. There was a substantial overlap between the TLR4inducible and NADPH oxidase-inducible genes, with 10 genes significantly upregulated 3-fold or more in both datasets (Fig. 1a, b, d). Many of the $S$. Typhimurium genes that respond to both TLR4 and NADPH oxidase protect against oxidative damage, including $a h p C F, d p s$ and sufABC (Zheng et al., 2001; Storz et al., 1989; Porwollik et al., 2003; Nachin et al., 2003; Lee et al., 2004; Janssen et al., 2003; Halsey et al., 2004). These data link the antibacterial effect of TLR4 in the phagosome with $\mathrm{NADPH}$ oxidase and are consistent with the literature suggesting a role for TLR4 in the regulation of this enzyme (Ryan et al., 2004; Pacquelet et al., 2007). 


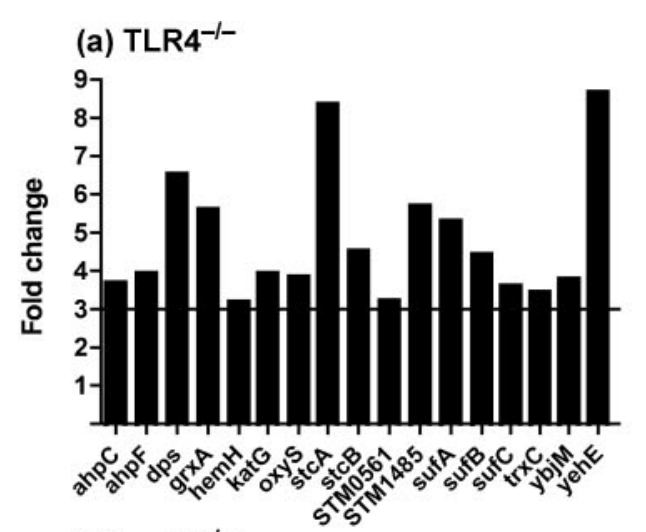

(b) gp $91^{-1-}$

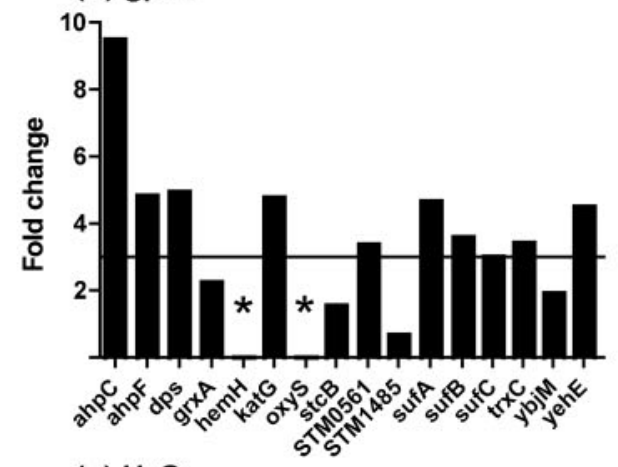

(c) $\mathrm{H}_{2} \mathrm{O}_{2}$

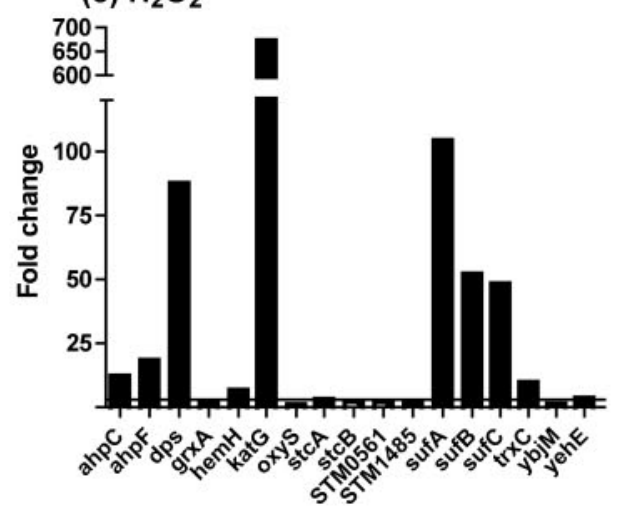

(d)

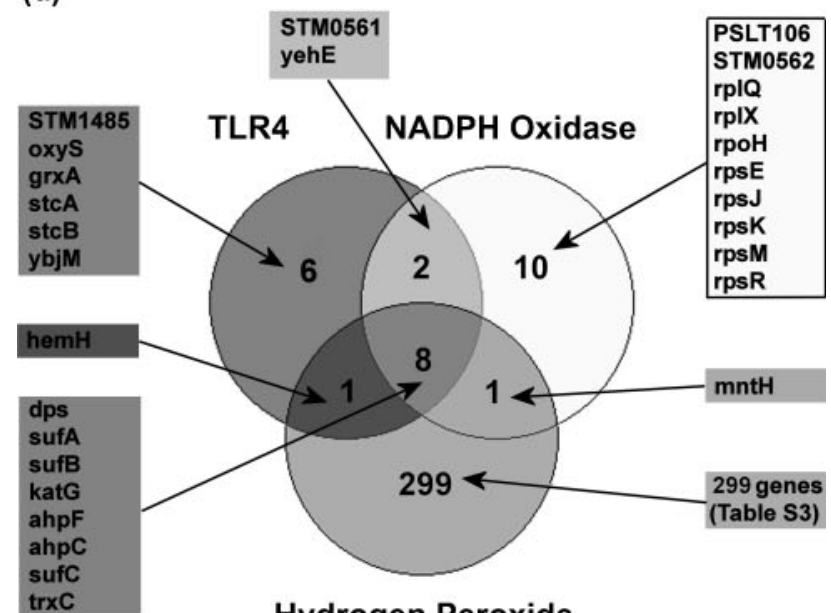

Hydrogen Peroxide
Fig. 1. (a) Expression levels, shown as fold-change, of $S$. Typhimurium genes significantly induced 3 -fold or more in BMDMs in the presence of TLR4 (compared to the TLR4 ${ }^{-1-}$ control; Supplementary Table S1). (b) Expression levels, shown as fold-change, of the TLR4-inducible $S$. Typhimurium genes in BMDMs in the presence of NADPH oxidase (compared to gp $91^{-1-}$ controls). Genes where expression values are missing, due to lack of signals on the microarrays, are indicated with an asterisk (Supplementary Table S2). (c) Expression levels, shown as foldchange, of the TLR4-inducible $S$. Typhimurium genes in response to treatment with $1 \mathrm{mM}$ hydrogen peroxide in LB broth (compared to untreated controls; Supplementary Table S3). The cut-off of a 3fold change is marked with a horizontal line in panels (a)-(c). (d) Venn diagram showing the overlap between genes significantly upregulated $>3$-fold in response to TLR4, NADPH oxidase or hydrogen peroxide.

\section{Identification of eight TLR4-inducible, NADPH oxidase-inducible genes that respond to peroxide in vitro}

We hypothesized that the 'TLR4-inducible' and 'NADPH oxidase-inducible' genes would contribute to resisting environmental stress. Many of the bactericidal effects of NADPH oxidase are mediated by hydrogen peroxide, making it a useful in vitro mimic of NADPH oxidase, albeit with limitations. We therefore determined whether the $21 \mathrm{NADPH}$ oxidaseinducible genes were also induced by hydrogen peroxide in vitro. $S$. Typhimurium was grown to mid-exponential phase and subjected to a short treatment with hydrogen peroxide. Expression of 309 and 428 genes was up- or downregulated more than 3-fold, respectively, in response to hydrogen peroxide (Supplementary Table S5). Twelve genes were 'NADPH oxidase-inducible', but not peroxide-inducible and eight genes were 'TLR4-inducible' but not peroxide-inducible (Fig. 1d and Supplementary Tables S1 and S2). In contrast, nine genes (ahpC, ahpF, kat G, dps, sufA, sufB, sufC, $\operatorname{trx} C$ and $m n t H$ ) were induced by both peroxide in vitro and intracellular NADPH oxidase during macrophage infection, and all but one $(m n t H)$ of these were also upregulated in the presence of TLR4 (Fig. 1b, c, d; Supplementary Table S1).

\section{Deletion of individual TLR4- and NADPH oxidase- inducible genes results in sensitivity of S. Typhimurium to oxidative stress in vitro}

We made deletion mutants of genes that were both NADPH oxidase-inducible and TLR4-inducible and characterized the resistance of the resulting mutants to hydrogen peroxide stress in vitro. We focused initially on the kat $G$, dps, sufA and STM0561 genes, which were all induced more than 3-fold in the presence of NADPH oxidase. The kat $G$, dps and sufA genes were also most strongly induced by hydrogen peroxide in vitro (673-, 104- and 88-fold, respectively; Supplementary Table S5). The addition of peroxide to the $S$. Typhimurium parent strain SL1344 caused an initial approximately 10-fold reduction of bacterial numbers from an initial inoculum of approximately $5 \times 10^{4}$ c.f.u. $\mathrm{ml}^{-1}$ and prevented bacterial 
growth for approximately $6 \mathrm{~h}$ (Fig. 2a). The four mutants showed substantial reductions in viable counts upon addition of hydrogen peroxide (Fig. 2b-e). The strains lacking katG or STM0561 were killed by exposure to hydrogen peroxide for $45 \mathrm{~min}$ (Fig. 2b, e). $S$. Typhimurium lacking $d p s$ was killed by 200 min of hydrogen peroxide challenge. In contrast, bacteria lacking sufA showed a rapid decrease in bacterial numbers over the initial $3 \mathrm{~h}$ of the experiment, followed by a stabilization to around $5 \times 10^{2}$ c.f.u. $\mathrm{ml}^{-1}$ over the next $5 \mathrm{~h}$ of the experiment (Fig. 2d). At $20 \mathrm{~h}$ post-challenge, this strain had managed to grow and had apparently entered stationary phase (data not shown).

\section{Individual TLR4- and NADPH oxidase-inducible genes are not required for virulence in mice}

In order to characterize the TLR4-inducible genes further, we investigated whether they are important for murine infection. We constructed additional deletions of the STM1485, oxyS, dps, grxA and yehE genes in $S$. Typhimurium. Deletions of multiple genes or operons were constructed; ahpCF, stcABCD and sufABCDSE, and the ahpCF and kat $G$ mutations were combined into a single strain (Table 1). In total 12 mutant strains, carrying deletions in 14 of the 17 TLR4-inducible genes, and 9 of the 10 genes that were both TLR4- and NADPH oxidaseinducible, were constructed. All the $S$. Typhimurium mutants grew similarly to the SL1344 wild-type in LB broth and on LB agar (data not shown). We infected C57BL/6 mice intravenously with each of the $S$. Typhimurium mutants. Only the $S$. Typhimurium $d p s$ mutant had reduced growth in spleen and liver (Fig. 3a-d). Lower spleen and liver viable counts compared with those seen using SL1344 were also observed after infection of gp91 ${ }^{-1-}$ mice with the $d p s$ mutant, suggesting that Dps contributes to resistance to other control mechanisms in
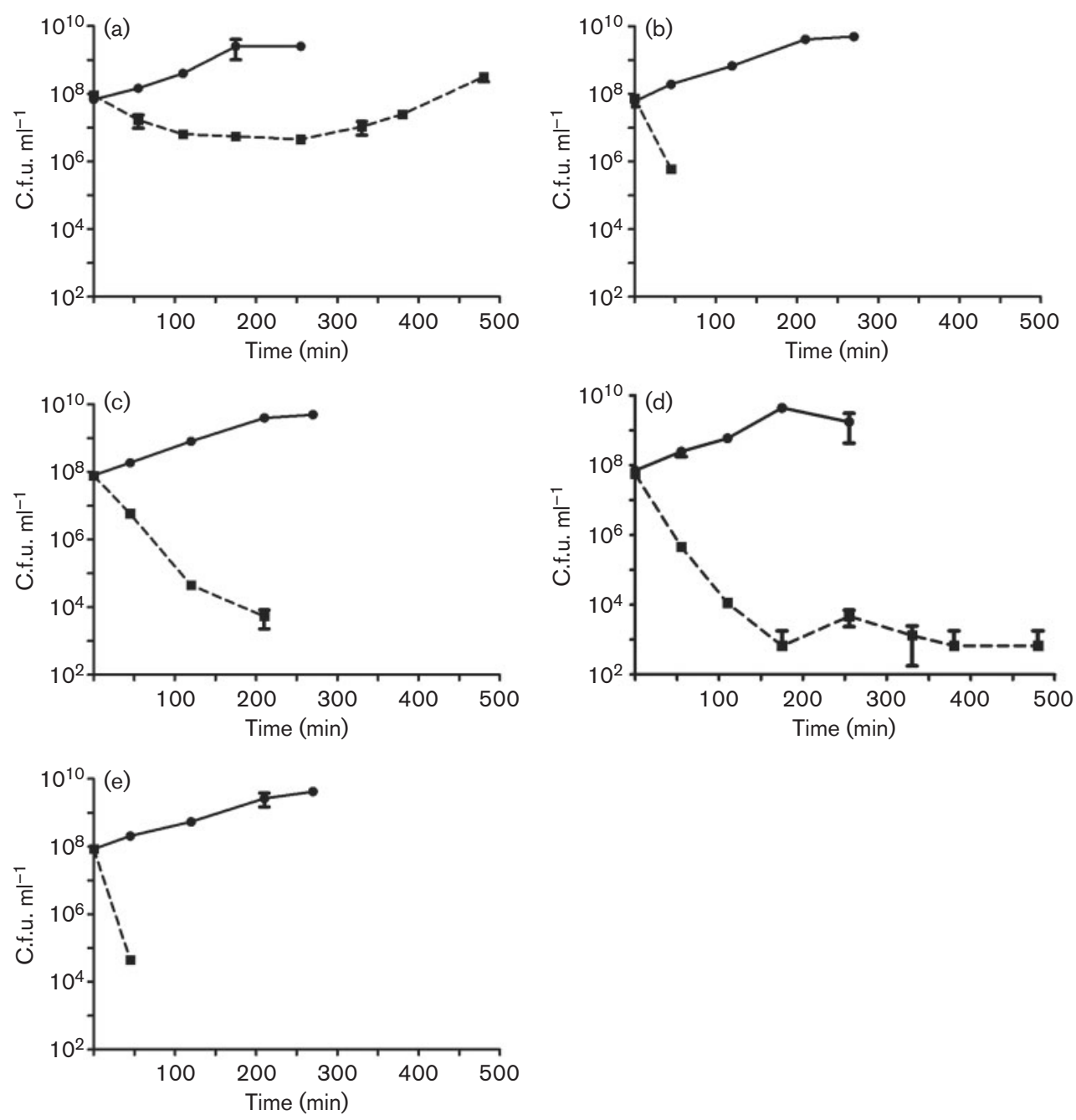

Fig. 2. Bacterial growth and viability of $S$. Typhimurium mutants in the presence $(\boldsymbol{\square})$ and absence $(\bullet)$ of $5 \mathrm{mM}$ hydrogen peroxide. (a) SL1344 (b) $\Delta k a t G$, (c) $\Delta d p s$, (d) $\Delta s u f A$, (e) $\Delta S T M 0561$. Error bars show standard deviation, and are too small to be visible in some cases. 

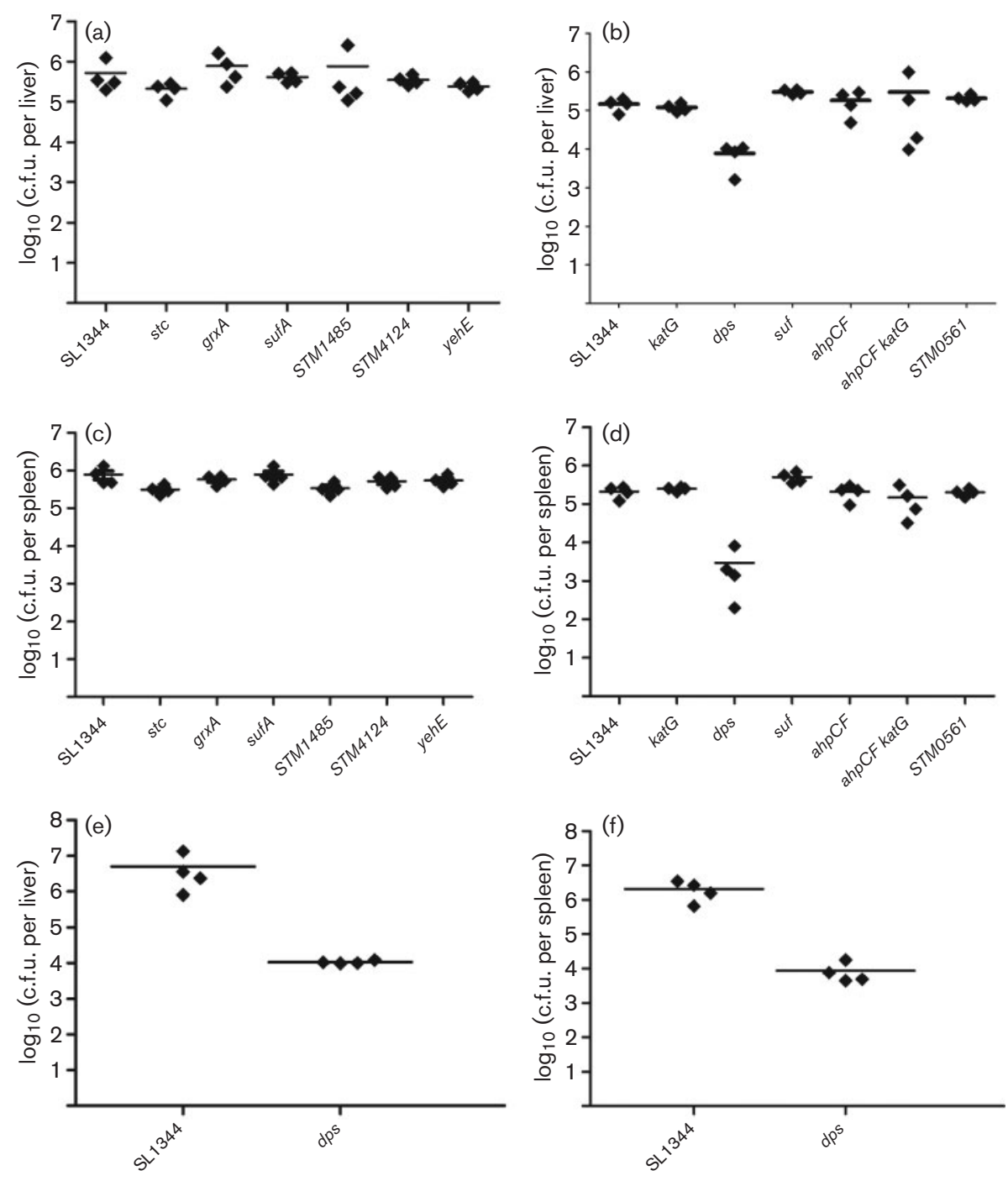

Fig. 3. ( $a-d)$ Bacterial counts in livers $(a, b)$ and spleens $(c, d) 3$ days after intravenous infection of C57BL/6 mice with $S$. Typhimurium SL1344 and strains with specific genes deleted. Data points represent individual infections. Each experiment was carried out two or three times; representative examples are shown. (e, $f$ ) Bacterial counts from livers (e) and spleens ( $f$ ) of gp $91^{-/-}$mice 3 days after intravenous infection with S. Typhimurium SL1344 and the dps mutant strain.

addition to oxidative stress (Fig. 3e, f). None of the other deletion mutants exhibited a significantly reduced bacterial load in either spleen or liver.

\section{DISCUSSION}

TLR4 is a crucial component of the murine innate immune response to $S$. Typhimurium. Although the signalling pathways downstream of this receptor are an area of intense research and are increasingly well characterized, the direct effects of TLR4 activation on a pathogen during infection have received little attention. We describe 'snapshot' transcriptomic profiling of $S$. Typhimurium during infection of primary murine macrophages as a method of characterizing the response of the pathogen to these conditions, and consequently to the intracellular environment encountered by $S$. Typhimurium inside macrophages. This novel approach is testing the hypothesis that bacteria can be used as 'sensors' to understand host defence mechanisms.

Through the comparison of $S$. Typhimurium gene expression in $\mathrm{TLR}^{-1-}$ and wild-type BMDMs, we identified 17 genes that were clearly induced in response to the effects of TLR4 signalling during macrophage infection. Several of these genes have been ascribed functions related to resistance to oxidative damage. These include $d p s, k a t G, a h p C$ and $a h p F$, and four components of the suf operon (Buchmeier et al., 1995; Halsey et al., 2004; Lee et al., 2008; Poole \& Ellis, 1996; Taylor et al., 1998). The induction of multiple genes known to protect $S$. 
Typhimurium against oxidative stress could reflect an intracellular environment rich in oxygen radicals, and suggests that TLR4 contributes to this environment.

There is a substantial degree of overlap between the $S$. Typhimurium genes induced in response to TLR4 and those induced in response to active NADPH oxidase (10 out of 17 TLR4-inducible genes). This suggests a functional link between these two components of the innate immune system. Our identification of eight genes induced in response to TLR4 and NADPH oxidase that also respond to hydrogen peroxide in vitro suggests that TLR4 contributes to oxygen radical generation in macrophages. The involvement of TLR4 in production of reactive oxygen species remains contentious. Previous experiments have shown that TLR4-deficient macrophages are able to elicit the same level of early killing of $S$. Typhimurium as wildtype peritoneal macrophages (Cook et al., 2007). This period corresponds to the 'NADPH oxidase-mediated' phase, and at $1 \mathrm{~h}$ after infection no measurable difference in hydrogen peroxide or superoxide was detected in TLR4deficient cells (O'Brien et al., 1980; Vazquez-Torres et al., 2004; Eisenstein et al., 1982). Peritoneal macrophages lacking the TLR adaptor protein, MyD88, exhibit impaired killing of $S$. Typhimurium and a deficiency in NADPH oxidase-mediated oxygen radical generation. This defect in production of reactive oxygen species was not observed in TLR4-deficient macrophages (Laroux et al., 2005). However, LPS is known to elicit a TLR4-dependent oxidative burst in THP-1 monocyte-like cells and BMDM (Ryan et al., 2004). More recent data have shown that IRAK-4, a regulatory kinase that operates downstream of TLR4, phosphorylates the $\mathrm{p} 47^{\text {phox }}$ subunit to activate NADPH oxidase in response to LPS in human neutrophils (Pacquelet et al., 2007). The data that we present here support the idea that there is a link between TLR4 signalling and NADPH oxidase activity in murine BMDMs.

We constructed defined deletion mutants of a number of NADPH oxidase-inducible genes and tested their hydrogen peroxide sensitivity in vitro, revealing that under these conditions katG, dps, sufA and STM0561 are apparently required for full resistance to peroxide-induced stress. For three of these four mutant strains (kat $G, d p s$ and STM0561), hydrogen peroxide was completely bactericidal, with no viable bacteria recovered after $200 \mathrm{~min}$ of challenge. These mutants were not complemented, and thus it cannot be definitively stated that the observed hydrogen peroxide sensitivity is due to deletion of these genes, and not some polar effect or secondary site mutation. However, in the case of the Dps- and catalasedeficient mutants, these results are consistent with published data (Buchmeier et al., 1995; Halsey et al., 2004). Deletion of the suf operon in Escherichia coli results in increased sensitivity to various superoxide generators but not to hydrogen peroxide, whereas hydrogen peroxide sensitivity was observed in S. Typhimurium suf mutants (Lee et al., 2008). STM0561 is a gene of unknown function; the inferred amino acid sequence has $74 \%$ identity to a 104 residue stretch of $E$. coli CusS, a putative two-component sensor histidine kinase involved in copper-inducible expression of a potential copper ion anti-porter (Munson et al., 2000). However, this identity only covers the histidine kinase domain of CusS, so while it is probably safe to assume that this protein is involved in bacterial signal transduction nothing can be assumed about what it is responding to in the environment.

The sensitivity of $S$. Typhimurium to hydrogen peroxide in vitro only mimics part of the oxidative stress that bacteria are exposed to in vivo. The ability of pathogens to resist oxidative stress involves multiple genes with redundant or complementary functions, and their role in Salmonella virulence has proven to be difficult to dissect. However, the role of three oxidative stress-responsive genes has been defined. The heat-shock protein HtrA is involved in resistance to oxidative stress in vitro, and htrA mutants are attenuated in mice but cause a lethal infection in animals lacking a functional NADPH oxidase (Mutunga et al., 2004). Thioredoxin 1, encoded by $\operatorname{tr} x A$, promotes intracellular replication and virulence of $S$. Typhimurium, but thioredoxin 2, encoded by $\operatorname{trxC}$, does not play a significant role during $S$. Typhimurium infection (Bjur et al., 2006). SodCI mutants lacking a $\mathrm{Cu} / \mathrm{Zn}$-superoxide dismutase are slightly attenuated in mice, as measured by reduced mortality after intraperitoneal infection in Nrampresistant mice. It should be noted however that the mutants were still able to kill approximately $50 \%$ of the genetically resistant mice infected at the dose used, indicating that there is no absolute requirement for SodC1 for murine infection with $S$. Typhimurium (Ammendola et al., 2008).

In this study, deletion of many of the TLR4- and NADPH oxidase-inducible genes, alone or in combination, did not cause significant attenuation in intravenous murine infections. Only deficiency in $d p s$ resulted in reduced bacterial loads in the spleens and livers of infected mice when compared to wild-type SL1344 infection. This lack of attenuation of mutants lacking oxidative stress response genes has also been observed elsewhere, for example with katG, ahpC and grxA (Boyer et al., 2002; Buchmeier et al., 1995; Taylor et al., 1998). No global mutagenesis approach has yet identified a role for individual oxidative stress response genes in murine infection by $S$. Typhimurium (Hensel et al., 1995; Lawley et al., 2006).

Our findings illustrate the strength of global gene expression profiling as a means to investigate the response of an intracellular pathogen to specific innate immune responses. This approach has revealed a complete and subtle set of information that illustrates the complexity of the bacterial response to host innate immunity. The response of $S$. Typhimurium to the innate immune mechanisms encountered within the phagosome is complex, and involves induction of numerous genes with seemingly redundant functions. This dataset would not have been arrived at if a simple single gene mutagenesis method had been used to investigate these complex 
pathways. Moreover, some of the genes identified clearly play a role in resisting other host resistance mechanisms. For instance, the mutants in the NADPH oxidaseresponsive genes exhibit strong peroxide sensitivity in vitro, yet only one, $d p s$, is significantly attenuated in vivo. Interestingly infection of gp91 $1^{-1-}$ mice with the $S$. Typhimurium $d p s$ mutant also resulted in recovery of reduced numbers of bacteria compared to the numbers recovered from infection with the wild-type $S$. Typhimurium strain, especially in the spleen, suggesting that Dps does not simply protect against NADPH oxidase. Dps also binds to and coats DNA (Almiron et al., 1992) and it is thought thus to protect DNA from damage in a non-specific fashion. This clearly illustrates that there are many complex elements to the host-pathogen interaction.

We conclude that $S$. Typhimurium induces a variety of genes in response to the activity of the potent TLR4- and $\mathrm{NADPH}$ oxidase-mediated innate immune mechanisms. Most of the induced genes do not contribute to virulence in isolation, but are likely to represent multiple redundant mechanisms involved in resisting these host resistance factors, suggesting that these mechanisms are important for survival and proliferation of $S$. Typhimurium within the host cell.

\section{ACKNOWLEDGEMENTS}

This work was funded by a BBSRC project grant (BB/C005503/1) to C.E. B. and D. J.M., a BBSRC core strategic grant to J.C.D.H., a Defra VTRI VT104 grant to T.H. and V.D. and an EC grant (SAFEHOUSE 423000E) to C. A.-A.

\section{REFERENCES}

Akira, S., Uematsu, S. \& Takeuchi, O. (2006). Pathogen recognition and innate immunity. Cell 124, 783-801.

Almiron, M., Link, A. J., Furlong, D. \& Kolter, R. (1992). A novel DNAbinding protein with regulatory and protective roles in starved Escherichia coli. Genes Dev 6, 2646-2654.

Ammendola, S., Pasquali, P., Pacello, F., Rotilio, G., Castor, M., Libby, S. J., Figueroa-Bossi, N., Bossi, L., Fang, F. C. \& Battistoni, A. (2008). Regulatory and structural differences in the $\mathrm{Cu}, \mathrm{Zn}$-superoxide dismutases of Salmonella enterica and their significance for virulence. J Biol Chem 283, 13688-13699.

Bjur, E., Eriksson-Ygberg, S., Aslund, F. \& Rhen, M. (2006). Thioredoxin 1 promotes intracellular replication and virulence of Salmonella enterica serovar Typhimurium. Infect Immun 74, 51405151.

Boyer, E., Bergevin, I., Malo, D., Gros, P. \& Cellier, M. F. (2002). Acquisition of $\mathrm{Mn}(\mathrm{II})$ in addition to $\mathrm{Fe}(\mathrm{II})$ is required for full virulence of Salmonella enterica serovar Typhimurium. Infect Immun 70, 6032-6042.

Breitling, R., Armengaud, P., Amtmann, A. \& Herzyk, P. (2004). Rank products: a simple, yet powerful, new method to detect differentially regulated genes in replicated microarray experiments. FEBS Lett 573, 83-92.

Buchmeier, N. A., Libby, S. J., Xu, Y., Loewen, P. C., Switala, J., Guiney, D. G. \& Fang, F. C. (1995). DNA repair is more important than catalase for Salmonella virulence in mice. J Clin Invest 95, 10471053.

Cook, P., Totemeyer, S., Stevenson, C., Fitzgerald, K. A., Yamamoto, M., Akira, S., Maskell, D. J. \& Bryant, C. E. (2007). Salmonella-induced SipBindependent cell death requires Toll-like receptor-4 signalling via the adapter proteins Tram and Trif. Immunology 122, 222-229.

Datsenko, K. A. \& Wanner, B. L. (2000). One-step inactivation of chromosomal genes in Escherichia coli K-12 using PCR products. Proc Natl Acad Sci U S A 97, 6640-6645.

Eisenstein, T. K., Deakins, L. W., Killar, L., Saluk, P. H. \& Sultzer, B. M. (1982). Dissociation of innate susceptibility to Salmonella infection and endotoxin responsiveness in $\mathrm{C} 3 \mathrm{HeB} / \mathrm{FeJ}$ mice and other strains in the C3H lineage. Infect Immun 36, 696-703.

Eriksson, S., Lucchini, S., Thompson, A., Rhen, M. \& Hinton, J. C. (2003). Unravelling the biology of macrophage infection by gene expression profiling of intracellular Salmonella enterica. Mol Microbiol 47, 103-118.

Eriksson-Ygberg, S., Clements, M. O., Rytkonen, A., Thompson, A., Holden, D. W., Hinton, J. C. D. \& Rhen, M. (2006). Polynucleotide phosphorylase negatively controls $s p v$ virulence gene expression in Salmonella enterica. Infect Immun 74, 1243-1254.

Faucher, S. P., Porwollik, S., Dozois, C. M., McClelland, M. \& Daigle, F. (2006). Transcriptome of Salmonella enterica serovar Typhi within macrophages revealed through selective capture of transcribed sequences. Proc Natl Acad Sci U S A 103, 1906-1911.

Fields, P. I., Swanson, R. V., Haidaris, C. G. \& Heffron, F. (1986). Mutants of Salmonella typhimurium that cannot survive within the macrophage are avirulent. Proc Natl Acad Sci U S A 83, 51895193.

Haas, A. (2007). The phagosome: compartment with a license to kill. Traffic 8, 311-330.

Halsey, T. A., Vazquez-Torres, A., Gravdahl, D. J., Fang, F. C. \& Libby, S. J. (2004). The ferritin-like Dps protein is required for Salmonella enterica serovar Typhimurium oxidative stress resistance and virulence. Infect Immun 72, 1155-1158.

Hensel, M., Shea, J. E., Gleeson, C., Jones, M. D., Dalton, E. \& Holden, D. W. (1995). Simultaneous identification of bacterial virulence genes by negative selection. Science 269, 400-403.

Hinton, J. C., Hautefort, I., Eriksson, S., Thompson, A. \& Rhen, M. (2004). Benefits and pitfalls of using microarrays to monitor bacterial gene expression during infection. Curr Opin Microbiol 7, 277-282.

Hoiseth, S. K. \& Stocker, B. A. (1981). Aromatic-dependent Salmonella typhimurium are non-virulent and effective as live vaccines. Nature 291, 238-239.

Hoshino, K., Takeuchi, O., Kawai, T., Sanjo, H., Ogawa, T., Takeda, K. \& Akira, S. (1999). Cutting edge: Toll-like receptor 4 (TLR4)-deficient mice are hyporesponsive to lipopolysaccharide: evidence for TLR4 as the lps gene product. J Immunol 162, 3749-3752.

Janssen, R., van der Straaten, T., van Diepen, A. \& van Dissel, J. T. (2003). Responses to reactive oxygen intermediates and virulence of Salmonella typhimurium. Microbes Infect 5, 527-534.

Jeffery, I. B., Higgins, D. G. \& Culhane, A. C. (2006). Comparison and evaluation of methods for generating differentially expressed gene lists from microarray data. BMC Bioinformatics 7, 359.

Jones, P. W., Dougan, G., Hayward, C., Mackensie, N., Collins, P. \& Chatfield, S. N. (1991). Oral vaccination of calves against experimental salmonellosis using a double aro mutant of Salmonella typhimurium. Vaccine 9, 29-34.

Kelly, A., Goldberg, M. D., Carroll, R. K., Danino, V., Hinton, J. C. \& Dorman, C. J. (2004). A global role for Fis in the transcriptional 
control of metabolism and type III secretion in Salmonella enterica serovar Typhimurium. Microbiology 150, 2037-2053.

Kim, H. M., Park, B. S., Kim, J. I., Kim, S. E., Lee, J., Oh, S. C., Enkhbayar, P., Matsushima, N., Lee, H. \& other authors (2007). Crystal structure of the TLR4-MD-2 complex with bound endotoxin antagonist eritoran. Cell 130, 906-917.

Laroux, F. S., Romero, X., Wetzler, L., Engel, P. \& Terhorst, C. (2005). Cutting edge: MyD88 controls phagocyte NADPH oxidase function and killing of gram-negative bacteria. J Immunol 175, 5596-5600.

Lawley, T. D., Chan, K., Thompson, L. J., Kim, C. C., Govoni, G. R. \& Monack, D. M. (2006). Genome-wide screen for Salmonella genes required for long-term systemic infection of the mouse. PLoS Pathog 2, e11.

Lee, J. H., Yeo, W. S. \& Roe, J.-H. (2004). Induction of the sufA operon encoding $\mathrm{Fe}-\mathrm{S}$ assembly proteins by superoxide generators and hydrogen peroxide: involvement of OxyR, IHF and an unidentified oxidant-responsive factor. Mol Microbiol 51, 1745-1755.

Lee, K. C., Yeo, W. S. \& Roe, J. H. (2008). Oxidant-responsive induction of the suf operon, encoding a Fe-S assembly system, through Fur and IscR in Escherichia coli. J Bacteriol 190, 8244-8247.

Lembo, A., Kalis, C., Kirschning, C. J., Mitolo, V., Jirillo, E., Wagner, H., Galanos, C. \& Freudenberg, M. A. (2003). Differential contribution of Toll-like receptors 4 and 2 to the cytokine response to Salmonella enterica serovar Typhimurium and Staphylococcus aureus in mice. Infect Immun 71, 6058-6062.

Lucchini, S., Liu, H., Jin, Q., Hinton, J. C. \& Yu, J. (2005). Transcriptional adaptation of Shigella flexneri during infection of macrophages and epithelial cells: insights into the strategies of a cytosolic bacterial pathogen. Infect Immun 73, 88-102.

McCollister, B. D. \& Vazquez-Torres, A. (2006). Interactions of $S$. enterica with phagocytic cells. In Salmonella Infections: Clinical, Immunological and Molecular Aspects, pp. 255-278. Edited by P. Mastroeni \& D. J. Maskell. Cambridge, UK: Cambridge University Press.

Mo, E., Peters, S. E., Willers, C., Maskell, D. J. \& Charles, I. G. (2006). Single, double and triple mutants of Salmonella enterica serovar Typhimurium $\operatorname{deg} P(h \operatorname{tr} A), \operatorname{deg} Q(h h o A)$ and $\operatorname{deg} S(h h o B)$ have diverse phenotypes on exposure to elevated temperature and their growth in vivo is attenuated to different extents. Microb Pathog 41, 174-182.

Monack, D. M., Raupach, B., Hromockyj, A. E. \& Falkow, S. (1996). Salmonella typhimurium invasion induces apoptosis in infected macrophages. Proc Natl Acad Sci U S A 93, 9833-9838.

Munson, G. P., Lam, D. L., Outten, F. W. \& O'Halloran, T. V. (2000). Identification of a copper-responsive two-component system on the chromosome of Escherichia coli K-12. J Bacteriol 182, 5864-5871.

Mutunga, M., Graham, S., De Hormaeche, R. D., Musson, J. A., Robinson, J. H., Mastroeni, P., Khan, C. M. \& Hormaeche, C. E. (2004). Attenuated Salmonella typhimurium htrA mutants cause fatal infections in mice deficient in NADPH oxidase and destroy NADPH oxidase-deficient macrophage monolayers. Vaccine 22, 4124-4131.

Nachin, L., Loiseau, L., Expert, D. \& Barras, F. (2003). SufC: an unorthodox cytoplasmic ABC/ATPase required for [Fe-S] biogenesis under oxidative stress. EMBO J 22, 427-437.

Nagy, G., Danino, V., Dobrindt, U., Pallen, M., Chaudhuri, R., Emody, L., Hinton, J. C. \& Hacker, J. (2006). Down-regulation of key virulence factors makes the Salmonella enterica serovar Typhimurium $r f a H$ mutant a promising live-attenuated vaccine candidate. Infect Immun 74, 5914-5925.

O'Brien, A. D., Rosenstreich, D. L., Scher, I., Campbell, G. H., MacDermott, R. P. \& Formal, S. B. (1980). Genetic control of susceptibility to Salmonella typhimurium in mice: role of the LPS gene. J Immunol 124, 20-24.
Pacquelet, S., Johnson, J. L., Ellis, B. A., Brzezinska, A. A., Lane, W. S., Munafo, D. B. \& Catz, S. D. (2007). Cross-talk between IRAK-4 and the NADPH oxidase. Biochem J 403, 451-461.

Paulin, S. M., Jagannathan, A., Campbell, J., Wallis, T. S. \& Stevens, M. P. (2007). Net replication of Salmonella enterica serovars Typhimurium and Choleraesuis in porcine intestinal mucosa and nodes is associated with their differential virulence. Infect Immun 75, 3950-3960.

Pollock, J. D., Williams, D. A., Gifford, M. A., Li, L. L., Du, X., Fisherman, J., Orkin, S. H., Doerschuk, C. M. \& Dinauer, M. C. (1995). Mouse model of X-linked chronic granulomatous disease, an inherited defect in phagocyte superoxide production. Nat Genet 9, 202-209.

Poole, L. B. \& Ellis, H. R. (1996). Flavin-dependent alkyl hydroperoxide reductase from Salmonella typhimurium. 1. Purification and enzymatic activities of overexpressed $\mathrm{AhpF}$ and $\mathrm{AhpC}$ proteins. Biochemistry 35, 56-64.

Porwollik, S., Frye, J., Florea, L. D., Blackmer, F. \& McClelland, M. (2003). A non-redundant microarray of genes for two related bacteria. Nucleic Acids Res 31, 1869-1876.

Rankin, J. D. \& Taylor, R. J. (1966). The estimation of doses of Salmonella typhimurium suitable for the experimental production of disease in calves. Vet Rec 78, 706-707.

Rosenberger, C. M., Scott, M. G., Gold, M. R., Hancock, R. E. \& Finlay, B. B. (2000). Salmonella typhimurium infection and lipopolysaccharide stimulation induce similar changes in macrophage gene expression. J Immunol 164, 5894-5904.

Royle, M. C., Totemeyer, S., Alldridge, L. C., Maskell, D. J. \& Bryant, C. E. (2003). Stimulation of Toll-like receptor 4 by lipopolysaccharide during cellular invasion by live Salmonella typhimurium is a critical but not exclusive event leading to macrophage responses. J Immunol 170, 5445-5454.

Ryan, K. A., Smith, M. F., Jr, Sanders, M. K. \& Ernst, P. B. (2004). Reactive oxygen and nitrogen species differentially regulate Toll-like receptor 4-mediated activation of NF-kappa B and interleukin-8 expression. Infect Immun 72, 2123-2130.

Santos, R. L., Zhang, S., Tsolis, R. M., Kingsley, R. A., Adams, L. G. \& Baumler, A. J. (2001). Animal models of Salmonella infections: enteritis versus typhoid fever. Microbes Infect 3, 1335-1344.

Schmieger, H. (1972). Phage P22-mutants with increased or decreased transduction abilities. Mol Gen Genet 119, 75-88.

Steele-Mortimer, O. (2008). The Salmonella-containing vacuole: moving with the times. Curr Opin Microbiol 11, 38-45.

Storz, G., Jacobson, F. S., Tartaglia, L. A., Morgan, R. W., Silveira, L. A. \& Ames, B. N. (1989). An alkyl hydroperoxide reductase induced by oxidative stress in Salmonella typhimurium and Escherichia coli: genetic characterization and cloning of ahp. J Bacteriol 171, 20492055.

Takeuchi, O., Hoshino, K., Kawai, T., Sanjo, H., Takada, H., Ogawa, T., Takeda, K. \& Akira, S. (1999). Differential roles of TLR2 and TLR4 in recognition of gram-negative and gram-positive bacterial cell wall components. Immunity 11, 443-451.

Taylor, P. D., Inchley, C. J. \& Gallagher, M. P. (1998). The Salmonella typhimurium AhpC polypeptide is not essential for virulence in $\mathrm{BALB} / \mathrm{c}$ mice but is recognized as an antigen during infection. Infect Immun 66, 3208-3217.

Teghanemt, A., Prohinar, P., Gioannini, T. L. \& Weiss, J. P. (2007). Transfer of monomeric endotoxin from MD-2 to CD14: characterization and functional consequences. J Biol Chem 282, 36250-36256.

Vazquez-Torres, A., Vallance, B. A., Bergman, M. A., Finlay, B. B., Cookson, B. T., Jones-Carson, J. \& Fang, F. C. (2004). Toll-like receptor 4 dependence of innate and adaptive immunity to 
Salmonella: importance of the Kupffer cell network. J Immunol 172, 6202-6208.

Villarreal-Ramos, B., Manser, J. M., Collins, R. A., Chance, V., Eckersall, D., Jones, P. W. \& Dougan, G. (2000). Susceptibility of calves to challenge with Salmonella typhimurium 4/74 and derivatives harbouring mutations in htrA or purE. Microbiology 146, 2775-2783.

Waterman, S. R. \& Holden, D. W. (2003). Functions and effectors of the Salmonella pathogenicity island 2 type III secretion system. Cell Microbiol 5, 501-511.
Weiss, D. S., Raupach, B., Takeda, K., Akira, S. \& Zychlinsky, A. (2004). Toll-like receptors are temporally involved in host defense. J Immunol 172, 4463-4469.

Zheng, M., Wang, X., Templeton, L. J., Smulski, D. R., LaRossa, R. A. \& Storz, G. (2001). DNA microarray-mediated transcriptional profiling of the Escherichia coli response to hydrogen peroxide. J Bacteriol 183, 4562-4570.

Edited by: D. L. Gally 